\title{
Isolation and sequencing of doublesex/male abnormal 3 (DM) related transcription factor (Dmrt) genes from the Asian toad Bufo gargarizans (Cantor, 1842)
}

\author{
Wen Chen, Liu-wang Nie and Ping-ping Zheng \\ Life Science College, Anhui Normal University, Wuhu, Anhui province,China.
}

\begin{abstract}
The doublesex/male abnormal 3 (dsx/mab-3 or DM) domain gene family involved in sexual development encodes putative transcription factors including a DNA-binding homology motif, the DM domain. We used highly degenerate primers to clone and sequence seven distinct DM related transcription factor (Dmrt) genes from the Asian toad (Bufo gargarizans Cantor, 1842). A database search for the cloned sequences revealed the following percentage identity with the homologous Dmrt genes of the human: BgDmrt1 $=97 \%$, BgDmrt2 $=97 \%$, three isoforms of BgDmrt3 $(B g D m r t 3 a=93 \%, B g D m r t 3 b=95 \%, B g D m r t 3 c=100 \%)$ and two isoforms of $B g D m r t 5(B g D m r t 5=97 \%$, $B g D m r t 5=91 \%)$. Based on DM domain amino acid sequence similarities we constructed a phylogenetic tree which grouped vertebrate and invertebrate Dmrt genes into seven distinct subfamilies. The DM domains of both human and the newly-discovered Bufo gargarizans genes contained two conserved zinc-chelating sites (CCHC and $\mathrm{HCCC})$, except BgDmrt3b, which contained the CCRC and HCCC sites.
\end{abstract}

Key words: Bufo gargarizans, Dmrt genes, DM domain, SSCP.

Received: July 26, 2006; Accepted: March 8, 2007.

The DM domain gene family has some members with a conserved DNA-binding DM domain encoding putative transcription factors related to the sexual regulators Doublesex (DSX) from Drosophila melanogaster and Male abnormal 3 (MAB-3) from Caenorhabditis elegans (Erdman and Burtis, 1993; Raymond et al., 1998). The DM domain has a highly intertwined structure that chelates two zinc atoms, and makes specific DNA contacts predominantly in the minor groove (Zhu et al., 2000). Doublesex and MAB-3 related transcription factor (Dmrt) genes have recently been cloned from a wide range of vertebrates, including fish, amphibians, reptiles, birds and mammals (Mastuda et al., 2002; Smith et al., 2002; Kettlewell et al., 2000; Shibata et al., 2002). These genes have been found to play essential roles in a variety of sex developmental processes, and at least some of these functions have been conserved. For example, Dmrtl gene knock-out mice have severe defects in testis differentiation and $D M Y$ mutant medaka fish (Oryzias latipes) show sex reversal during which XY fish carrying a mutant DM-domain gene on the Y chromosome (the $D M Y$ gene) develop not as males but as females (Raymond et al., 2000; Mastuda et al., 2002). The

Send correspondence to Liu-wang Nie. Life Science College, Anhui Normal University, 1 East Beijing Road, Wuhu, 241000, Anhui, China. E-Mail: Iwnie@mail.ahnu.edu.cn. frog (Rana rugosa) Dmrt1 gene is expressed in the differentiating testis but is not detectable during ovarian differentiation (Shibata et al., 2002). The zebrafish (Danio rerio) Terra/Dmrt2 gene is an early left-sided expressed gene that links left-right patterning with bilateral synchronization of the segmentation clock (Saude et al., 2005). The Dmrt3 gene is primarily expressed in the forebrain, neural tube and nasal placode of both mice and chickens (Smith et al., 2002), while the expression of Dmrt5 in adult zebrafish is restricted to brain cells and developing germ cells (Guo et al., 2004).

The widely distributed Asian toad (Bufo gargarizans Cantor, 1842), also called the Chusan Island toad, plays an important ecological role in the environments where it lives. Although Shang (1983) showed that Bufo gargarizans has a ZZ/ZW type chromosomal sex determination system, the molecular mechanisms of sex determination and differentiation remain unclear for this species. As a prelude to understanding the involvement of Dmrt genes in the sexual development of this toad, we cloned the DM domain gene family of $B$. gargarizans using degenerate primers from genomic DNA. In the present paper we report the cloning and nucleotide sequencing of seven different Dmrt genes from B. gargarizans. The phylogenetic relationships between the DM domain genes of verte- 
brates and invertebrates were also examined based on DM domain amino acid sequences.

We obtained three female and two male $B$. gargarizans from Wuhu city (Anhui province, China) and humanly sacrificed them. Total genomic DNA was isolated from muscle tissues by routine protocols (Sambrook et al., 1989). To amplify the conserved DM domains we used a pair of degenerate primers D1 (5'-TGCG(AGC)(AC)G(A G)TGC(AC)G(AG)AA(CT)CACGG-3') and D2 (5'-C (GT)(GC)AG(GC)GC(GC)ACCTG(GC)GC(AGCT)GCC AT-3') (Ren et al., 2001), the expected amplification fragment length being $140 \mathrm{bp}$ with these primers. The polymerase chain reaction (PCR) was carried out in a $25 \mu \mathrm{L}$ reaction mixture containing $50 \mathrm{ng}$ of genomic DNA, $1.5 \mathrm{mM} \mathrm{Mg}^{2+}$, $200 \mu \mathrm{M}$ of each dNTP, $0.2 \mu \mathrm{M}$ of each primer and 1 unit of Taq DNA polymerase (Promega, Peking, China). The cycling conditions were as follows: 32 cycles of $30 \mathrm{~s}$ at $94^{\circ} \mathrm{C}$, $40 \mathrm{~s}$ at $62{ }^{\circ} \mathrm{C}$ and $40 \mathrm{~s}$ at $72{ }^{\circ} \mathrm{C}$, followed by 7 min elongation at $72{ }^{\circ} \mathrm{C}$. The PCR products were cloned using the pMD 18-T Vector (TaKaRa, Dalian, China) and transformed into Escherichia coli strain DH5 $\alpha$ (Sangon, Shanghai, China) and 115 white clones were transferred to a plate of clones from an initial culture plate of lysogeny broth (LB) media containing 5-bromo-4-chloro-3-indolyl- $\beta$-Dgalactopyranoside (X-gal) and isopropyl- $\beta$-D-thiogalactoside (IPTG) and 75 positive clones with insert PCR fragment were confirmed using colony PCR (Shen et al., 2000). The distinctive positive clones were screened using single-strand conformation polymorphism (SSCP) (Nie et al., 1999) and sequenced using the universal sequencing primer on an ABI377 auto-sequencer (Applied Biosystems). All DNA sequences were analyzed using the basic local alignment search tool (BLAST) and CLUSTAL X1.8 programs and the phylogenetic tree was constructed using the molecular evolutionary genetic analysis (MEGA) program V2.1 (Kumra et al., 2001).

Using the $B$. gargarizans genomic DNA as template we obtained a 140 bp PCR fragment and we obtained seven distinct positive clones from the DNA of the male and female toads, although there was no difference between the clones in respect to the sex of the toads from which the clones originated (Figure 1).

Database searches and phylogenetic analysis of the clones uncovered seven unreported Dmrt sequences, representing distinct genes, which we named by adding the prefix Bg (Bufo gargarizans) and submitted to GenBank as Bufo gargarizans doublesex and mab -3 related transcription factor (BgDmrt) genes. The genes and their GenBank accession numbers and sequence identity to homologous human DMRT genes (in parentheses) are: BgDmrt1 (DQ217562， 97\%), BgDmrt2 (DQ217563， 97\%), BgDmrt3a (DQ217564, 93\%), BgDmrt3b (DQ217565, 95\%), BgDmrt3c (DQ217566, 100\%), BgDmrt5a (DQ217569, 97\%) and BgDmrt5b (DQ217571, 91\%). Note that $B g D m r t 3$ has three isoforms and Dmrt5 has two iso- forms. The putative amino acid sequences of the BgDmrt genes and the alignment between corresponding human $D M R T$ sequences are shown in Figure 2. The DM domains of both human and the newly-discovered B. gargarizans genes contained two conserved zinc-chelating sites ( $\mathrm{CCHC}$ and HCCC), except $B g D m r t 3 b$, which contained the CCRC and HCCC sites. It is probable that the DM domain genes act as a transcription regulator in sex determination and other developmental processes, mediated by the DNAbinding zinc motif (Zhu et al., 2000).

The putative amino acid sequences of the seven clones were compared with 34 published Dmrt gene sequences accessible at the NCBI BLAST server (Table 1), the DMdomain neighbor-joining phylogenetic tree being shown in

\begin{tabular}{|c|c|}
\hline & 40 \\
\hline $\mathrm{BgDmrt3b}$ & TGCGCCAGATGCOGGAATCAOGGGGTOCTCTOCTGGCTCAAGGGCCGCAAGCGCTATTGC \\
\hline BgDmrt3c & ....GC.G........................... \\
\hline BgDmrt3a & 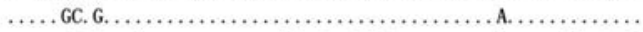 \\
\hline BgDmrt5a & 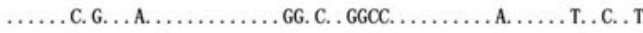 \\
\hline BgDmrt5b & 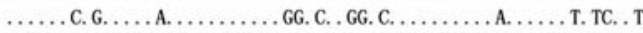 \\
\hline BgDmrt2 & $\ldots \ldots$ GC.G..A.A. $\ldots \ldots \ldots \ldots$ G.A. $\ldots$ C. $\ldots \ldots \ldots$ A. A. . T. T $\ldots$ T \\
\hline \multirow[t]{2}{*}{ BgDmrtl } & ..... AC. G. ............. CTATTCT. . TCCT. . G. .... T. A...... T. TC. . T \\
\hline & 100 \\
\hline $\mathrm{BgDm}$ (3b & CGCTTCAAGGATTGCACTTGTGAGAGGTGCATCCTGATCATTGAGAGGCAGCGGGTGATG \\
\hline BgDmrt3c & 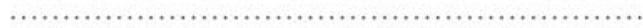 \\
\hline BgDmrt3a & 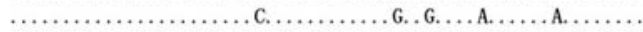 \\
\hline BgDmrt5a & 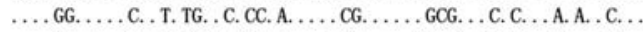 \\
\hline $\mathrm{BgDm}$ m5b & ....GG. . A. . C. . TCTG. . C. OC. A. ...COG. ....GGCG. . C. G. . A. . . . . \\
\hline BgDmrt2 & 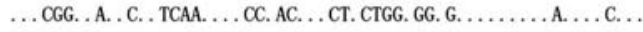 \\
\hline BgDmrt1 & 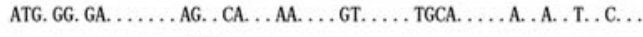 \\
\hline & 140 \\
\hline BgDmrt3b & GOGGCGCAGGTGGCCCTCOG \\
\hline $\mathrm{BgDm} r \mathrm{r} 3 \mathrm{c}$ & .T. C....C. .G..GA. \\
\hline BgDmrt3a & $\ldots$ C..C....C. . . ... \\
\hline BgDmrt5a & .............. \\
\hline $\mathrm{BgDm} r \mathrm{t} \mathrm{b}$ & ....C.......... \\
\hline BgDmrt2 & .C..C....C.G.GG. \\
\hline BgDmrt1 & .T. . C....C. . . G. \\
\hline
\end{tabular}

Figure 1 - The nucleotide sequences of seven doublesex/male abnormal 3 (dsx/mab-3 or DM) related transcription factor $(D M R T)$ gene DM domains from Bufo gargarizans $(B g)$. Dots indicate identities with BgDmrt3b.

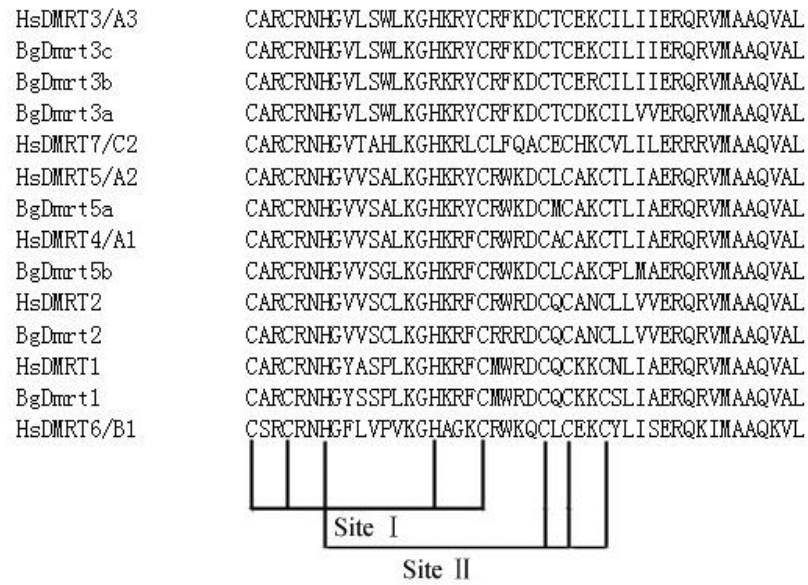

Figure 2 - Multiple alignment of the amino acid sequences of the doublesex/male abnormal 3 (dsx/mab-3 or DM) domains from toad (Bufo gargarizans or $\mathrm{Bg}$ ) and human (Homo sapiens or Hs) DM related transcription factors (DMRTs). Two DM domain Zn-chelating sites, SiteI (CCHC) and siteII (HCCC), are also shown. 
Table 1 - Doublesex/male abnormal 3 (dsx/mab-3 or DM) related transcription factor (DMRT) domain sequences included in this analysis.

\begin{tabular}{|c|c|c|c|}
\hline $\begin{array}{l}\text { DM domain sequence } \\
\text { (synonym) and species* }\end{array}$ & GenBank number & $\begin{array}{l}\text { DM domain sequence } \\
\text { (synonym) and species* }\end{array}$ & GenBank number \\
\hline Human & & Animal (continued) & \\
\hline DMRT1 & NM-021951 & Dmrt4 & \\
\hline DMRT2 & AF130729 & M. musculus & AF542047 \\
\hline DMRT3 (DMRTA3) & NM-021240 & E. brenchleyi & AAY26899 \\
\hline DMRT4 (DMRTA1) & AJ290954 & (Dmo) O. niloticus & AAF79932 \\
\hline DMRT5(DMRTA2) & AJ301580 & & \\
\hline DMRT6 (DMRTB1) & AJ291671 & Dmrt5 & \\
\hline \multirow[t]{2}{*}{ DMRT7 (DMRTC2) } & AJ291669 & M. musculus & AY145837 \\
\hline & & E. brenchleyi & AAY26900 \\
\hline Animal & & O. latipes & BAD00703 \\
\hline \multicolumn{4}{|l|}{ Dmrt1 } \\
\hline Mus musculus & NM-015826 & Dmrt6 & \\
\hline Gallus gallus & AF211349 & M. musculus & AF542048 \\
\hline Oncorhynchus mykiss & AAG17544 & & \\
\hline Eremias brenchleyi & AAY26895 & Dmrt7 & \\
\hline Takifugu rubripes & CAC42778 & M. musculus & AF542046 \\
\hline \multirow[t]{2}{*}{ (DMY)Oryzias latipes } & $\mathrm{AB} 071534$ & & \\
\hline & & F10C1.5 & \\
\hline Dmrt2 & & Caenorhabditis elegans & AAA93409 \\
\hline M. musculus & AF539811 & & \\
\hline Oreochromis niloticus & AA074518 & Dmrt99B & \\
\hline Chrysemys scripta elegans & AAG15567 & Drosophila melanogaster & NP_524549 \\
\hline T. rubripes & CAC42780 & & \\
\hline \multirow[t]{2}{*}{ (Terra) Danio rerio } & AF080622 & Dmrt11E & \\
\hline & & D. melanogaster & NM_078591 \\
\hline \multicolumn{4}{|l|}{ Dmrt3 } \\
\hline M. musculus & AAN77230 & Mab-3 & \\
\hline G. gallus & XP427822 & C. elegans & $\dagger$ \\
\hline T. rubripes & AJ295039 & & \\
\hline O. latipes & AAL02164 & & \\
\hline
\end{tabular}

*M. musculus $=$ mouse; G. gallus $=$ chicken; $O$. mykiss $=$ trout E. brenchleyi $=$ a wall lizard; $T$. rubripes $=$ Japanese pufferfish; $O$. latipes $=$ medaka fish; $O$. niloticus $=$ tilapia; $C$. s. elegans $=$ red-eared turtle; $D$. rerio $=$ zebrafish; $C$. elegans $=$ nematode; $D$. melanogaster $=$ fruit fly.

†Raymond et al., 1998.

Figure 3. Phylogenetic analysis showed that all these Dmrt genes were clustered into seven different subfamilies (Dmrt1-7), and confirmed the identity of the BgDmrt genes identified by us. However, the $B g D m r t 5 b$ was not included in the Dmrt 5 group, implying more accumulation of amino acid substitutions in $B g D m r t 5 b$ than in other members of the Dmrt 5 cluster. We also compared four different invertebrate DM domain proteins with other vertebrate DM domain proteins using Mab-3 from the nematode Caenorhabditis elegans as the outgroup and found that Dmrt2 was related to Dmrt11E from the fly Drosophila melanogaster, Dmrt4 was related to Drosophila melanogaster Dmrt99B and Dmrt5 was related to
Caenorhabditis elegans F10C1.5, suggesting that both the Dmrt2 and Dmrt4/Dmrt5 groups are ancient because they both contain invertebrate sequences. Ottolenghi et al. (2002) used structural analysis to show that the human genes DMRTA1, DMRTA2 and DMRTA3, also called DMRT4, DMRT5 and DMRT3 respectively (the alternative nomenclature for human $D M R T$ genes follows Volff et al. (2003), likewise for DMRTB1 and DMRTC2, which was called DMRT6 and Dmrt7 respectively), all have a DM domain and a DMRTA domain, phylogenetic analysis showing a relationship between DMRTA1 and DMRTA2 while DMRTA3 was more distant. Our neighbor-joining analysis supported a relationship between vertebrate Dmrt4 and 


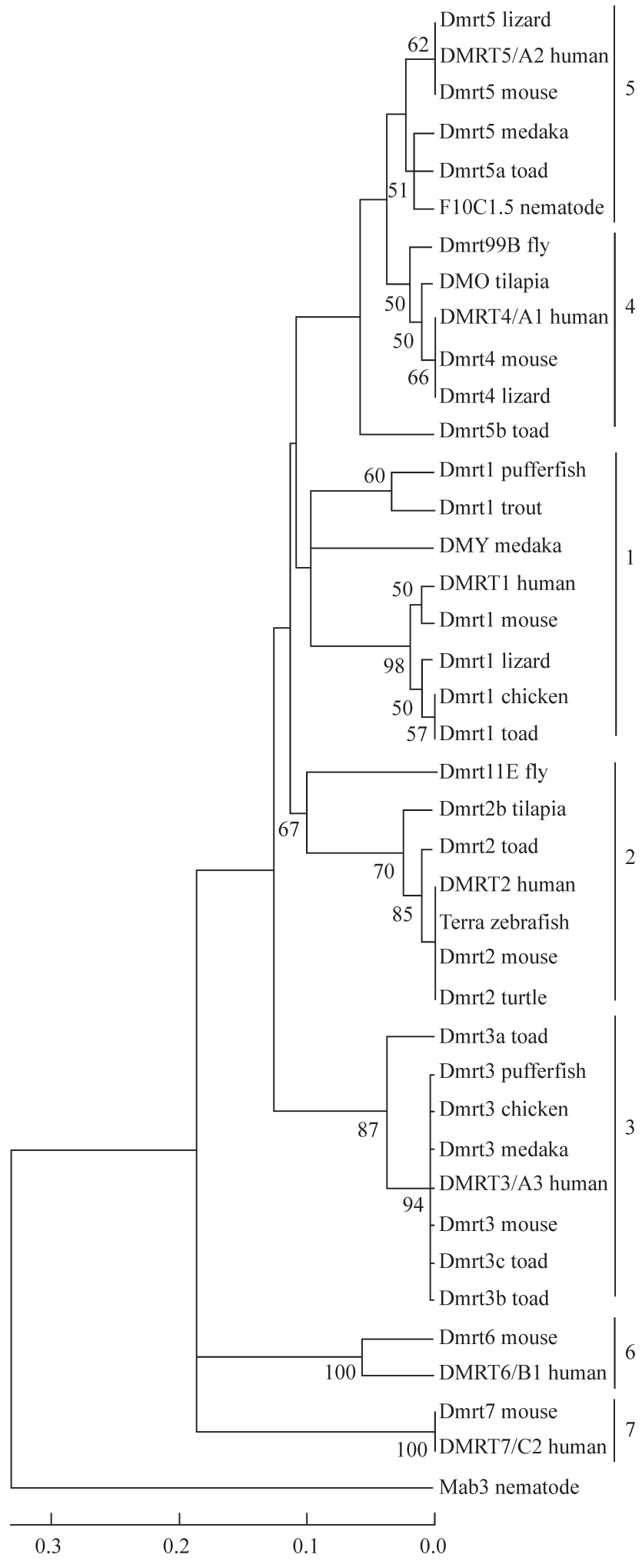

Figure 3 - Neighbor-joining phylogenetic tree (1000 replicates) of some vertebrate and invertebrate doublesex/male abnormal 3 (dsx/mab-3 or $\mathrm{DM})$ related transcription factor $(D M R T)$ gene family members based on amino acid sequence data. Nematode Mab-3 sequences were the outgroup. Branches with less than 50\% support have been collapsed. The toad DM domain sequences in the tree were the Bufo gargarizans sequences obtained during the research described in this paper.
Dmrt 5 but, in contrast, no closer phylogenetic relationship could be detected between Dmrt3 and Dmrt4/Dmrt5, although this does not preclude a close relationship. Volff et al. (2003) have pointed out that different Dmrt genes can be under very different evolutionary constraints. In our analysis the short branches in the Dmrt3 group suggests very strong sequence conservation, while the fact that the branches in the Dmrt1 group appear to be longer indicates much more relaxed evolutionary constraints for these sequences.

This is the first report describing Dmrt genes from $B$. gargarizans. Several of the clones obtained in this study indicate that Dmrt genes that are duplicated in B. gargarizans are present in mammals as single copies. For example, the human genome contains single copy of DMRT3 (DMRTA3), whereas this gene is present in at least three copies in B. gargarizans. Intriguingly, the BgDmrt3a gene is divergent from other Dmrt3 genes in the phylogenetic tree and the zinc-chelating site I of BgDmrt $3 b$ is cystine, cystine, arginine, cystine (CCRC) not cystine, cystine, histidine, cystine (CCHC). According to Prince and Pickett (2002), gene duplication is a mechanism by which new gene functions may be acquired. The very recent duplication of the Dmrtl has apparently led to the formation of the master male-determining $D M Y$ gene in the medaka fish, and similar scenarios might have generated new paralogs of other Dmrt genes in different taxa (Mastuda et al., 2002; Volff et al., 2003). The Dmrt gene duplication in B. gargarizans may have been the result of such a process, unlike the situation in teleost fish, which may have been the result of the ancient duplication of the whole genome. However, how these Dmrt genes function in the sexual development $B$. gargarizans still needs further experimental exploration.

\section{Acknowledgments}

This work was supported by the National Natural Science Fund of China (No.30640048) and Found of the Key Lab. of Biotic Environment and Ecology Safety in Anhui Province (2006). We thank two anonymous reviewers and the technical editor for critically reviewing the manuscript.

\section{References}

Erdman SE and Burtis K C (1993) The Drosphila doublesex proteins share a novel zinc finger related DNA binding domain. EMBO J 12:527-535.

Guo Y, Li Q, Gao S, Zhou X, He Y, Shang X, Cheng H and Zhou R (2004) Molecular cloning, characterization, and expression in brain and gonad of Dmrt5 of zebrafish. Biochem Biophys Res Commun 324:569-75.

Kettlewell JR, Raymond CS and Zarkower D (2000) Temperature-dependent expression of turtle Dmrt1 prior to sexual differentiation. Genesis 26:174-182. 
Kumra SK, Tamura K, Jakobsen IB and Nei M (2001) MEGA2: Molecular evolutionary genetics analysis software. Bioinformatics 17:1244-1245.

Matsuda M, Nagahama Y, Shinomiya A, Sato T, Matsuda C, Kobayashi T, Morrey CE, Shibata N, Asakawa S, Shimizu $\mathrm{N}$, et al. (2002) DMY is a Y-specific DM-domain gene required for male development in the medaka fish. Nature 417:559-563.

Nie LW, Shan XN and Guo CW (1999) The PCR amplification and SSCP analysis of Sox gene in turtles (Platysternon megacephalum and Cistoclemmys flavomarginatus). China J Appl Environ Biol 5:378-381.

Ottolenghi C, Fellous M, Barbieri M and McElreavery K (2002) Novel paralogy relations among human chromosome support a link between the phylogeny of doublesex-related genes and the evolution of sex determination. Genomics 79:333-343.

Prince VE and Pickett FB (2002) Splitting pairs: The diverging fates of duplicated genes. Nat Rev Genet 3:827-837.

Raymond CS, Murphy MW, O'Sullivan MG, Bardewell VJ and Zarkower D (2000) Dmrt1, a gene related to worm and fly sexual regulator, is required for mammalian testis differentiation. Genes Dev 14:2587-2595.

Raymond CS, Shamu CE, Shen MM, Seifert KJ, Hirsch B, Hodgkin J and Zarkower D (1998) Evidence for evolutionary conservation of sex-determining genes. Nature 391:691-695.

Ren LL, Cheng HH, Guo YQ and Zhou RJ (2001) Evolutionary conservation of Dmrt gene family in amphibians, reptiles and birds. Chinese Sci Bull 46:1992-1995.
Sambrook J, Friston EF and Maniatis T (1989) Molecular Cloning, a Laboratory Mannual. Cold Spring Harbor Laboratory Press, New York.

Saude L, Lourenco R, Goncalves A and Palmeirim I (2005) Terra is a left-right asymmetry gene required for left-right synchronization of the segmentation clock. Nat Cell Biol 7:918-20.

Shang KG (1983) Cytological evidence for ZZ/ZW sex-determination of Bufo bufo gagarizans. Acta Genetica Sinica 10:298-305

Shen GX, Zhu HF, Zhang Y, Wang S, Zhu ZG and Zhou C (2000) Screening of transfected bacteria by bacteria colonies PCR and plasmid PCR. Immunol J 2:149-151.

Shibata K, Takase M and Nakamura M (2002) The Dmrt1 expression in sex-reversed gonads of amphibians. Gen Comp Endocr 1127:232-41.

Smith CA, Hurley TM, McClive PJ and Sinclair AH (2002) Restricted expression of Dmrt3 in chicken and mouse embryos. Gene Expr Patterns 2:69-72.

Volff JN, Zarkower D, Bardwell VJ and Schartl M (2003) Evolutionary dynamics of the DM domain gene family in metazoans. J Mol Evol 57:241-249.

Zhu L, Wilken J, Phillips NB, Narendra U, Chan G, Stratton SM, Kent SB and Weiss MA (2000) Sexual dimorphism in diverse metazoans is regulated by a novel class of intertwined zinc fingers. Genes Dev 14:1750-1764.

\section{Internet Resources}

BLAST at http://www.ncbi.nlm.nih.gov/BLAST/.

CLUSTAL X1.8 at http://www.igh.cnrs.fr/bin/clustalxguess.cgi. Associate Editor: Louis Bernard Klaczko 